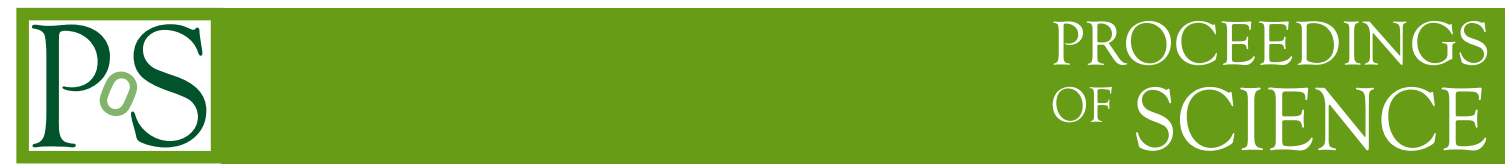

\title{
Spin Filtering at COSY - First Results
}

\author{
Dieter Oellers*, for the PAX-Collaboration \\ Uni Ferrara, FZ-Jülich \\ http://www2.fz-juelich.de/ikp/pax/portal/ \\ E-mail: di.oellersefz-juelich.de
}

In autumn 2011, a spin filtering experiment was performed at Jülich-COSY using a stored proton beam at $T_{p}=49.3 \mathrm{MeV}$. The equipment and procedures for the method to produce stored polarized beams was sucessfully commissioned and are established. After the idea of spin filtering is described, the experimental setup, the measurement cycle and first online results are presented.

8th International Conference on Nuclear Physics at Storage Rings-Stori11,

October 9-14, 2011

INFN, Laboratori Nazionali di Frascati, Italy

* Speaker. 


\section{Spin Filtering}

The PAX-Collaboration ${ }^{1}$ is investigating spin dependent attenuation as a method to produce polarized antiproton beams. In 1993 this method has been shown to work with protons at the FILTEX in Heidelberg [1].

During spin filtering a stored beam sufferes spin dependent attenuation by multiple traversing of a polarized internal gas target (Fig. 1 - left panel). More particles of one state than the other are scattered out of the ring acceptance and a polarized beam remains. The theoretical prediction for the difference $\sigma_{1}$ of the two spin dependent cross sections $\sigma_{1}=\sigma_{\uparrow \uparrow}-\sigma_{\uparrow \downarrow}$ in $p p$ scattering is plotted versus the kinetic beam energy in Fig. 1 - right panel.
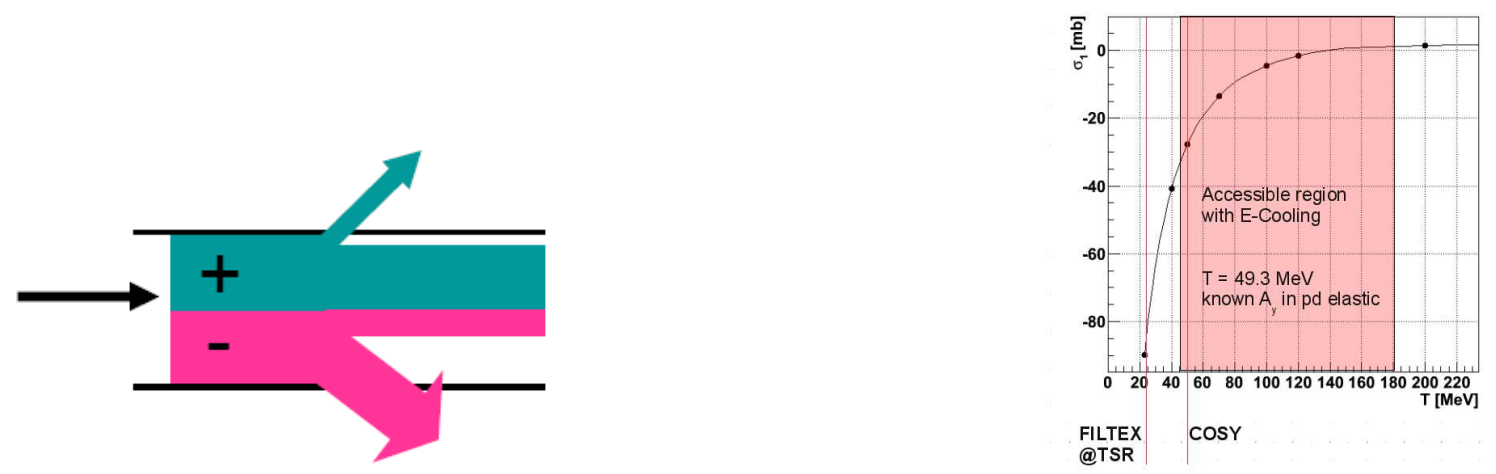

Figure 1: Left: If a stored beam of spin-1/2 particles looses more particles of one state than the other, the remaining beam gains polarization. Right: Theoretical calculations for the spin dependent cross-section $\sigma_{1}=\sigma_{\uparrow \uparrow}-\sigma_{\uparrow \downarrow}$.

\section{Experimental Setup}

In autumn 2011, a spin filtering experiment was performed at Jülich-COSY using a stored proton beam at $T_{p}=49.3 \mathrm{MeV}$ [2]. The overview of the COSY ring, showing the various components of the experimental setup used in the measurement is given in Fig. 2 -left panel. The experimental setup installed at the PAX interaction point at COSY is presented in the middle and right panel of Fig. 2.

In the following the performance of the different components of the experimental setup and the measurements that have been performed are described. Particular focus lies on

- the polarized target,

- the COSY ring performance,

- the beam polarimeter, and

- spin-filtering measurements.

\section{Polarized target section}

\subsection{Polarized target performance}

A schematic target layout of the PAX polarized target is presented in Fig. 3 - left panel [3].

${ }^{1}$ Polarized Antriproton eXperiments 

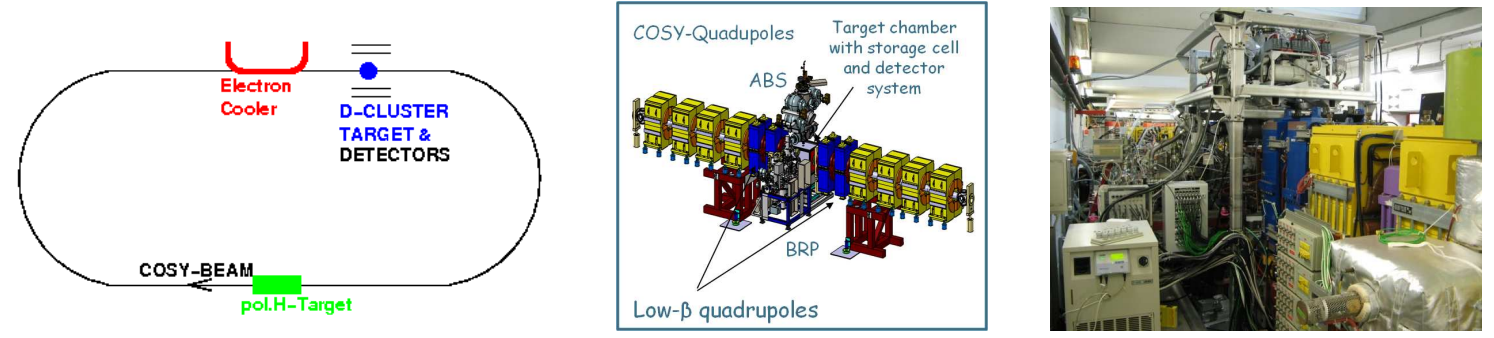

Figure 2: Left: The PAX polarized target is located in the straight section of the COSY ring opposite to the electron cooler, the RF solenoid of the spin-flipper is located in one of the arcs, its use is further clarified in the text of the report. Next to the electron cooler the detector setup for the beam polarimetry is shown. Middle panel: The PAX installation at the COSY ring comprises four additional quadrupole in order to provide a small $\beta$-function at the location of the storage cell. The Atomic beam source (ABS) and the BreitRabi target polarimeter and gas analyzer (BRP) are installed at the PAX target chamber. Right panel: Picture of the PAX installation at COSY.

The polarized target and its polarimeter performed smoothly during the whole data taking period.
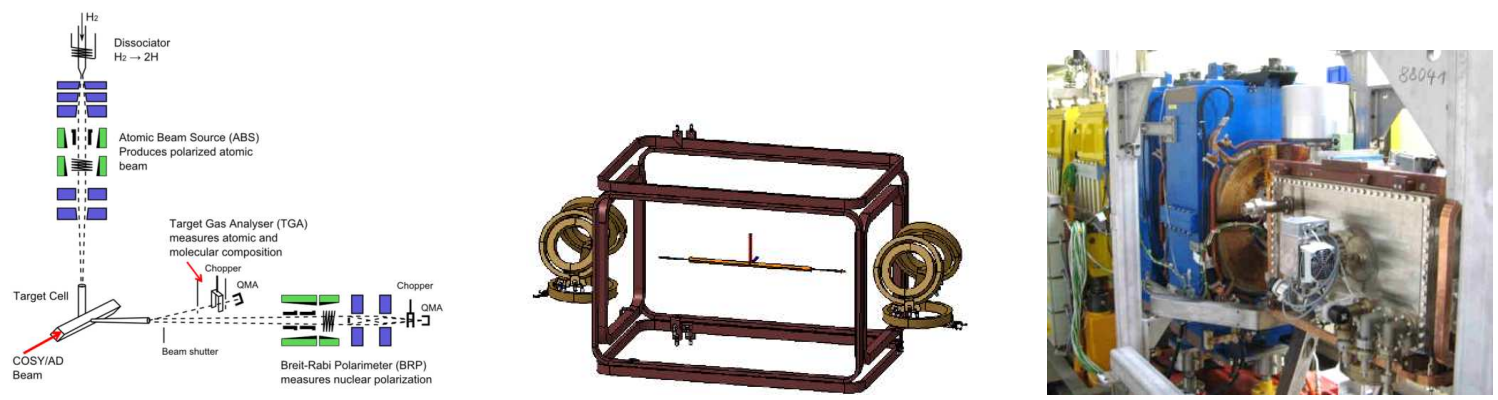

Figure 3: Left: Schematic layout of the PAX polarized target with the ABS in the vertical position, the storage cell on the beam axis and the BRP in the horizontal position. The Target Gas Analyzer (TGA) determines the atomic to molecular fraction of the effusive beam from the storage cell. Middle panel: holding field coil configuration mounted on the target chamber frame together with the compensating coils in front and behind the target chamber. The proper powering of the holding field and of the compensation coils allows for the orientation of the target holding field along any of the axes. During the measurement only the upper and lower coils have been powered so that the target holding field resulted in the vertical direction, hence the target polarization during the filtering experiments pointed either in $+y$ or in $-y$ direction. Right panel: picture of the target chamber: the coils are visible along the edges of the chamber.

In particular, the density in the target cell was measured through the observed beam deceleration induced by the target gas detected by the Schottky signal and resulted in a value larger than $5 \cdot 10^{13}$ atoms $/ \mathrm{cm}^{2}$ for one injected hyperfine state from the ABS. This value is consistent with expectations calculated from the known ABS flux $\left(3 \cdot 10^{16}\right.$ atoms/s) and the known conductance of the storage cell. During data taking, the target polarization was continuously monitored by the Breit-Rabi polarimeter and the Target Gas Analyzer, and the value was found to be constant in time and larger than $Q_{y}=0.7$. The magnetic field coils mounted at the chamber provided the vertical weak holding field $(B \approx 10 \mathrm{G})$, required to define the quantization axis for the target polarization (see Fig. 3 middle and right panel). The use of compensation coils in front and behind the main holding field coils allowed for an almost perfect compensation of the beam displacement: when powering 
the holding field coils no transverse displacement of the beam position could be detected by beam position monitors.

\subsection{Target chamber vacuum}

During the 2011 summer shutdown, a new NEG pumping system, designed and constructed in the FZJ mechanical workshops, has been installed below the PAX target chamber and put into operation. The pump has been realized by a battery of 10 NEG cartridges. During the activation of the cartridges at a temperature up to $450 \mathrm{C}$, a mechanical shutter separating the pump from the chamber was closed (see Fig. 4, right panel). In this way, the temperature in the target chamber was limited to less than about $80 \mathrm{C}$. By means of a calibrated $H_{2}$ gas inlet a pumping of $120001 / \mathrm{s}$ has been measured, according to design specifications.

The use of the pump during COSY operation allowed to achieve a target chamber pressure in the $10^{-10}$ mbar range without gas load from the ABS and in the low $10^{-8}$ mbar range with $\mathrm{H}$ gas injected from the ABS in one hyperfine state $\left(3 \cdot 10^{16} \mathrm{H}\right.$ atoms/s).
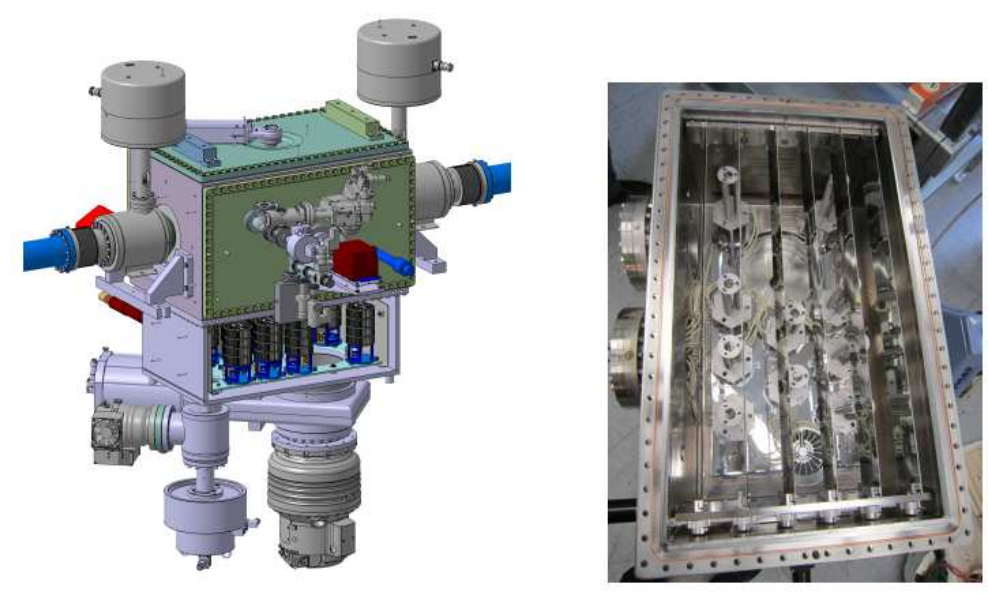

Figure 4: Left panel: schematic view of the target chamber on top of the newly installed NEG pump. Left and right to the target chamber the fast closing shutter valves can be seen as requested by the CERN vacuum regulation. Right panel: inner view of the NEG pump. The NEG cartridges are mounted on the bottom of the chamber; the opened mechanical shutter is clearly visible.

\section{COSY ring performance}

\subsection{Beam lifetime}

The newly installed NEG pump below the target chamber together with the activation of the neighbouring NEG coated tubes (produced at CERN) provided excellent vacuum conditions in the COSY ring (see Fig.5). The combination of the good vacuum and the effect of the low- $\beta$ section at the PAX interaction point produced a situation in which almost no effect on the beam-lifetime could be detected after injection of the polarized gas in the storage cell of the chamber. The same vacuum configuration is planned to be installed at CERN. 


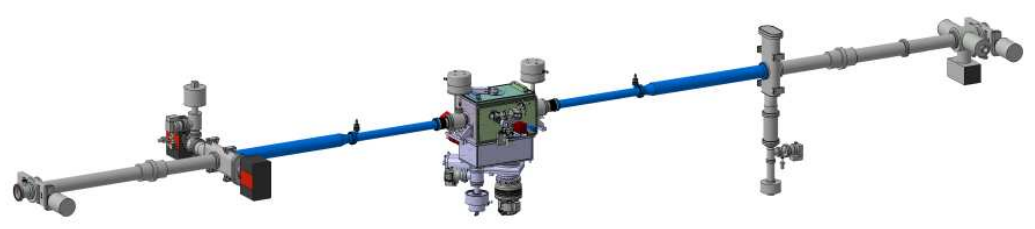

Figure 5: Overall view of the vacuum setup at the PAX interaction point. The NEG coated tubes are indicated in blue. The measured beam-lifetime during the spin-filtering cycles was $>6000$ s, exceeding the expectations and allowing for longer spin-filtering cycles than initially planned.

\subsection{Beam polarization lifetime and spin-flipper efficiency}

The polarization lifetime of the COSY beam has been measured prior to the spin-filtering experiments. The measurement has been performed by injecting a polarized proton beam into COSY and comparing the polarization at the beginning of a fill and after the beam was stored in the ring for $5000 \mathrm{~s}$ (see Fig. 6, left panel). The polarization loss during this time amounted to about $5 \%$, resulting in a polarization lifetime $\left(2.0 \cdot 10^{5} \pm 5 \cdot 10^{4}\right) \mathrm{s}$, confirming the prediction that no depolarizing resonances are present in the neighbourhood of the chosen machine tunes. In view of the polarization build-up experiment, the beam polarization lifetime can be considered as infinite.

In order to reduce systematic errors, spin-flips of the beam polarization have been introduced at every measurement cycle (as described below). The spin-flipper employs a resonant RF-solenoid and is part of the standard equipment of the COSY ring (see Fig. 2, upper panel). Before the measurement, the spin-flipper has been tuned to the proper working conditions and its efficiency has been measured in dedicated runs. A polarized proton beam was injected in the ring and its polarization was measured; subsequently, the spin-flipper has been switched on and a total number of 99 spin-flips have been induced on the beam. The remaining polarization of the beam has then been measured (Fig. 6, right panel). From the comparison between the initial and final polarization of the beam, the spin-flipper efficiency has been determined as $\varepsilon_{S F}=0.9887 \pm 0.001$.

\subsection{Beam polarimeter}

The beam polarization has been measured by means of the polarimeter installed at the ANKE interaction point in the opposite straight section of COSY (see Fig. 2). Beam polarimetry has been accomplished by making use of the analyzing power in proton-deuteron elastic scattering. For this purpose two three-layer silicon detectors [4] have been employed to detect the elastically scattered particles in the interaction between the stored proton beam and the ANKE deuterium cluster target (Fig. 7, left panel) [5]. Silicon layers of 70, 300, and 5000 $\mu \mathrm{m}$ thickness have been used for the realization of each telescope. The telescopes were symmetrically placed with respect to the target and longitudinally positioned in order to optimize the figure of merit $\left(F O M=d \sigma / d \Omega A_{y}^{2}\right)$ of the reaction (Fig. 7, right panel). The energy of the experiment has been chosen to be $49.3 \mathrm{MeV}$, since at this energy a precise determination of the beam polarization is possible, because accurate analyzing power data are available.

Elastically scattered deuterons and protons were clearly identified by the deposited energy in the different layers(see Fig. 8). Due to the low energy of the reaction, no background for deuterons 

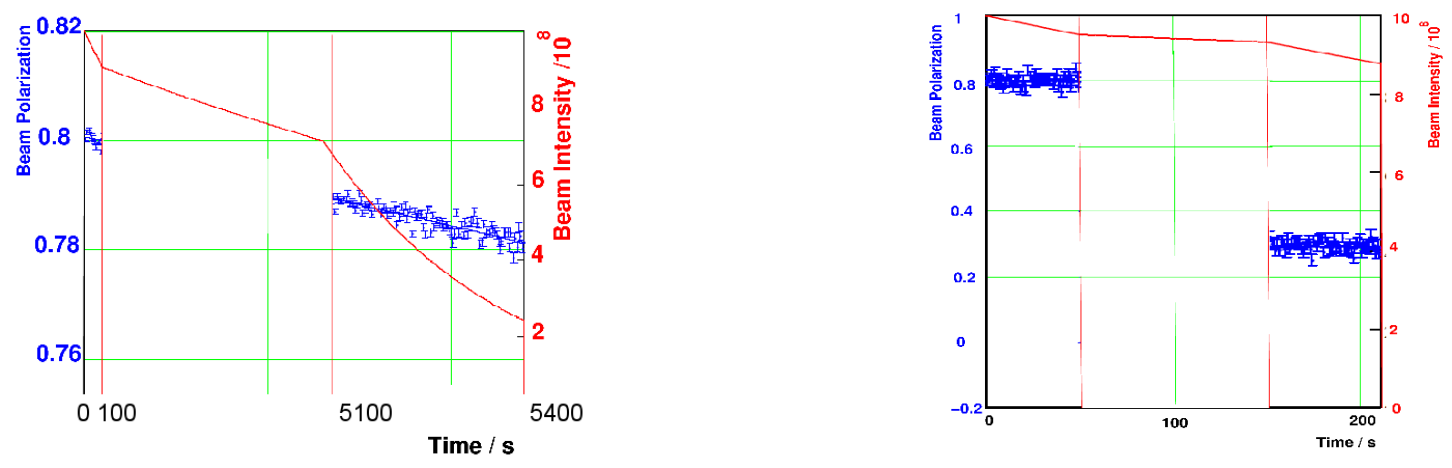

Figure 6: Left panel: measurement of the beam polarization lifetime. A polarized proton beam has been injected in the ring and its polarization measured at injection and after a storing period of $5000 \mathrm{~s}$ in the ring. From the comparison of the two values the beam polarization lifetime could be deduced. Right panel: measurement of the spin-flipper efficiency. The spin-flipper efficiency has been determined by injecting a polarized beam and comparing its polarization at injection and after 99 induced spin-flips.
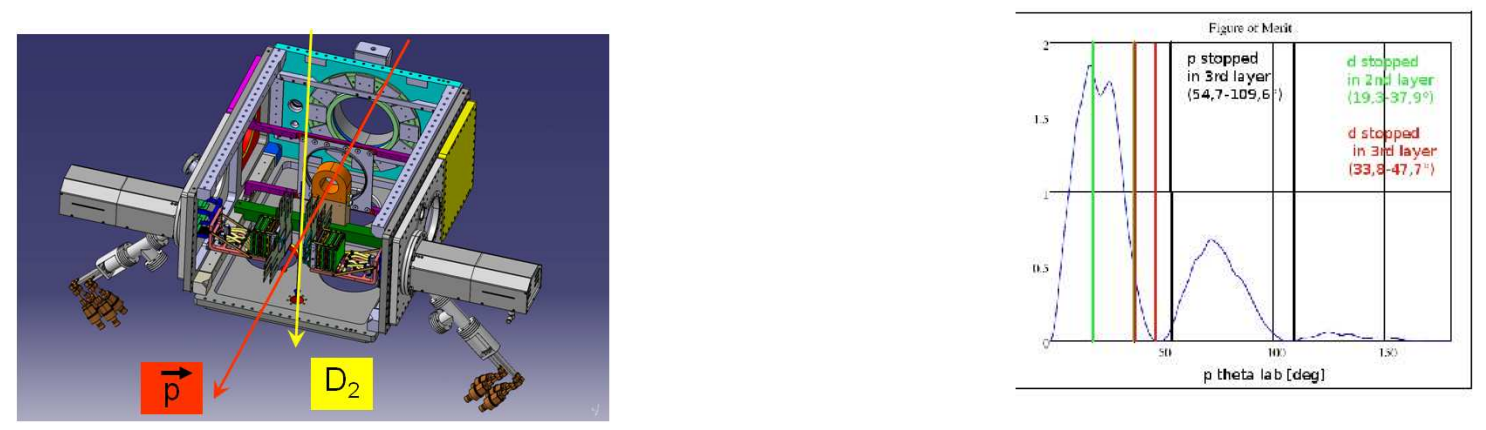

Figure 7: Left panel: Experimental setup for the measurement of the beam polarization as it was used during the beam time. The cluster target beam comes from the top and crosses the polarized proton beam stored in the machine. Elastically scattered deuterons are detected two silicon telescopes. Right panel: FOM for the polarized of the elastic reaction as a function of the deutoron scattering angle. Both $p$ and $d$ ejectiles are detected in the telescope system.

is expected, while some (small) background coming from deuteron breakup is expected for protons.

\section{Spin-filtering measurements}

\subsection{Spin-filtering cycle}

Typical spin filtering cycles are represented in Fig. 9 - left panel. The sequence of operations in a spin-filtering cycle is as follows:

- The unpolarized proton beam is injected in the COSY ring at $45 \mathrm{MeV}$. The beam is cooled and accelerated to an energy of $49.3 \mathrm{MeV}$ for the measurement. As mentioned already, the experimental energy has been chosen because of the existing data of the analyzing power. The typical number of particles injected and accelerated for every cycle was $5 \cdot 10^{9}$; 

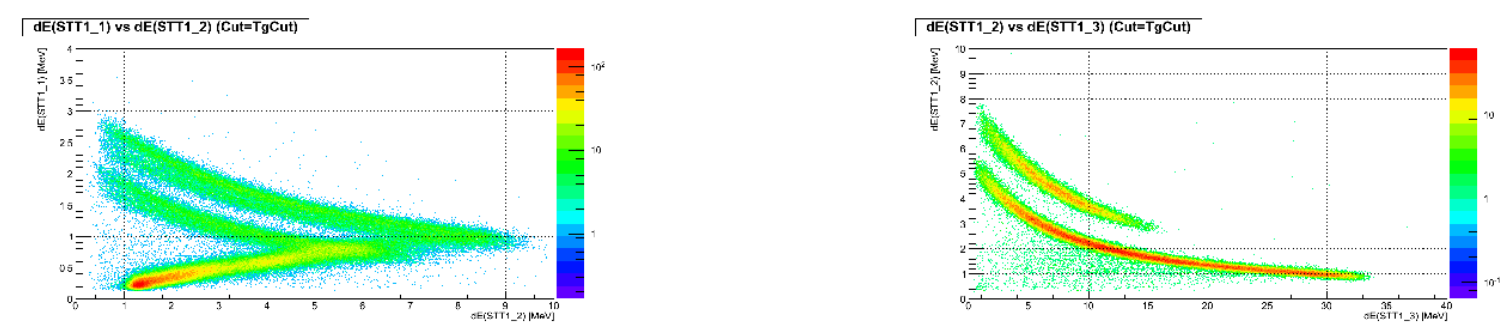

Figure 8: Proton and deuteron identification using the silicon telescope system. Left panel: deposited energy in the second $v s$ first layer. Right panel: deposited energy in the third $v s$ the second layer.

- At this point the spin-filtering cycle starts. Polarized gas is injected into the storage cell at the PAX interaction point and the holding field coils are powered. The typical duration of a spinfiltering cycle was 2 beam lifetimes, corresponding to a total spin-filtering time of $16000 \mathrm{~s}$. Note that the cycle shown in Fig. 9 - left panel - is actually shorter reflecting the expectations for the beam lifetime. Due to the excellent performance of the COSY ring during the beam time, the cycle was prolongated. An experimental cycle taken from an online monitor from the COSY control room is shown in Fig. 9 - right panel;

- At the end of the spin-filtering period, the PAX polarized target was switched off, and the ANKE deuterium cluster target and the data acquisition of the beam polarimeter were started. During the beam polarization measurement the beam polarization has been reversed twice by using the spin-flipper. This allows the determination of the induced beam polarization for every cycle, thereby reducing systematic errors. The total duration of the polarization measurement was $18500 \mathrm{~s}$;

- Spin-filtering cycles have been repeated for different directions of the target holding fields.
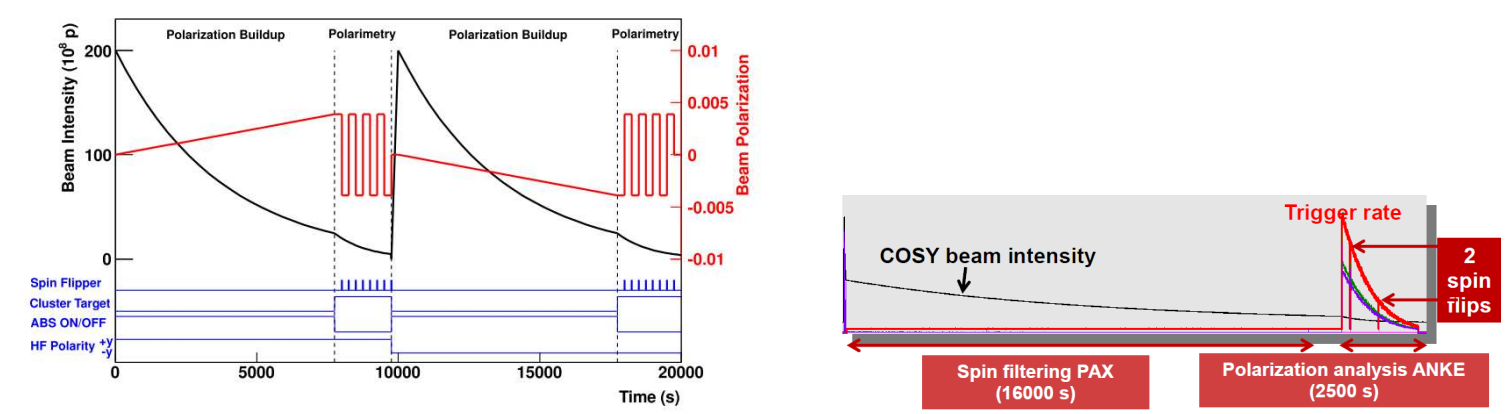

Figure 9: Left panel: Schematic representation of a typical spin-filtering cycle. The black curves represent the beam current, while the red ones show the polarization, induced in the beam. While the beam current decreases, the polarization in the beam builds up. At the end of the spin-filtering cycle the spin-flipper was switched on twice to allow for the measurement of the polarization. In the picture, two cycles for different orientations of the target holding field are indicated. Right panel: Picture taken from an online monitor of the COSY control room representing a spin-filtering cycle. At the end of the cycle the trigger rate can be noticed together with the two points in time where the spin flipper was activated. 


\subsection{Zero measurement}

In order to provide a zero polarization calibration of the detector, a series of cycles without spin-filtering has been carried out in addition. To be as close as possible to the experimental conditions of a standard filtering cycle, the zero-measurement cycle reflected exactly the same sequence of operations, differing only in the number of injected particles $\left(<1 \cdot 10^{9}\right)$ and the duration of the spin-filtering part (180s). A total number of 98 cycles of this type have been collected, so that the acquired statistics is twice as large as the one of the spin-filtering measurements.

\section{Analysis}

At the end of the beam time, analysis of the acquired data has started. The analysis is performed by two separate groups in order to cross-check each step, which comprise:

- Calibration of the STT

- Analysis of the "Null"-measurement

- Investigation of systematic errors

- Analysis of the spin-filtering measurements

- Determination of a precise spin-dependent cross-section

\section{Conclusion}

In the period August to October 2011, the PAX Collaboration has performed a spin-filtering test experiment with protons at the COSY storage ring. All the subsystems of the experimental setup (polarized target, COSY ring and related equipment, beam-polarimeter) worked as expected or even better, like for the vacuum system resulting in very long beam lifetimes. Particularly the new NEG pump system installed below the target chamber was very effective for the achievement of the ideal experimental conditions. A series of spin-filtering cycles has been prepared and successfully accomplished. A careful analysis of the experimental data is ongoing.

\section{References}

[1] F. Rathmann et al., New Method to Polarize Protons in a Storage Ring and Implications to Polarize Antiprotons, PRL 71, 1379 (1993).

[2] D. Oellers et al., Beam Request http://www2.fz-juelich.de/ikp/pax/portal/documents/proposals/files/beamrequest20110318.pdf, (2011).

[3] A. Nass et al., The HERMES polarized atomic beam source, Nucl. Instr. Meth. A505, 633 (2003).

[4] R. Schleichert et al., A self-triggering Silicon Tracking Telescope for Spectator Proton Detection, IEEE (2001).

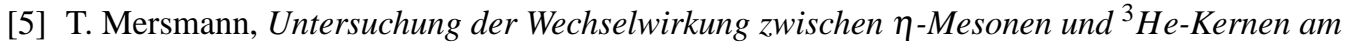
Experimentaufbau ANKE, Doktorabeit, Institut für Kernphysik, Westfälische Wilhelms-Universität Münster (2007). 